\title{
Using Planned Adaptation to Implement Evidence-Based Programs with New Populations
}

\author{
Shawna J. Lee · Inna Altschul · Carol T. Mowbray
}

Published online: 29 February 2008

(C) Springer Science+Business Media, LLC 2008

\begin{abstract}
The Interactive Systems Framework (ISF) for Dissemination and Implementation (Wandersman et al. 2008) elaborates the functions and structures that move evidence-based programs (EBPs) from research to practice. Inherent in that process is the tension between implementing programs with fidelity and the need to tailor programs to fit the target population. We propose Planned Adaptation as one approach to resolve this tension, with the goal of guiding practitioners in adapting EBPs so that they maintain core components of program theory while taking into account the needs of particular populations. Planned Adaptation is a form of capacity building within the Prevention Support System that provides a framework to guide practitioners in adapting programs while encouraging researchers to provide information relevant to adaptation as a critical aspect of dissemination research, with the goal of promoting wider dissemination and better implementation of EBPs. We illustrate Planned Adaptation using the JOBS Program (Caplan et al. 1989), which was developed for recently laid-off, working- and middle-class workers and subsequently implemented with welfare recipients.
\end{abstract}

S. J. Lee $(\square)$

School of Social Work and Merrill-Palmer Skillman Institute for Child and Family Development, Wayne State University, 4756 Cass Avenue, Detroit MI, USA

e-mail: shawnal@wayne.edu

I. Altschul

Graduate School of Social Work, University of Denver,

Denver, CO, USA

e-mail: inna@du.edu

C. T. Mowbray

School of Social Work \& Department of Psychology,

University of Michigan, Ann Arbor MI, USA
Keywords Evidence-based practice - Dissemination · Preventive intervention Program theory

In recent years there has been an increased emphasis on the use of evidence-based programs (EBPs) in social service settings (e.g., Fraser 2004; Gira et al. 2004; Howard et al. 2003). EBPs are "interventions for which there is consistent scientific evidence showing that they improve client outcomes" (Drake et al. 2001: 180). The use of EBPs in practice settings is highly desirable for both the client and the service provider (Rosen 2003). Yet, EBPs are not often offered in service settings, and when EBPs are provided there are a number of challenges to their successful implementation.

One reason for the lack of effective implementation of EBPs in practice settings is the considerable gap between prevention science and intervention practice. Once a program has been tested effective in a research setting, there are few mechanisms to facilitate its dissemination to the wider community or to support individual, organizational, and community capacity for program implementation. The ISF (Wandersman et al. 2008) addresses this challenge by providing a broad framework expressly for the purpose of moving knowledge from science to practice. A key question within the ISF is how to build practitioner capacity to support the implementation of EBPs. We address this question by proposing a mechanism to guide practitioners in the process of adapting programs to new contexts, and we point to the need for researchers to elucidate critical program components that should guide program adaptation.

\section{Program Adaptation as a Dissemination and Implementation Problem}

In most models of prevention science successful implementation of a program is defined by lack of modification to 
manualized content (Elliott and Mihalic 2004), and it is well established that fidelity is important to maintain program outcomes (Blakely et al. 1987; Mowbray et al. 2003). However, the standard of strict adherence to manualized protocols may not be practical in many social service settings, particularly when there is little ongoing outside program monitoring (Fagan and Mihalic 2003). Service providers generally seek to serve a population rather than to test a theoretical model (Backer 2001), and thus often wish to adapt programs to directly address the needs or characteristics of their clients (e.g., Bernal and Sáez-Santiago 2006; Castro et al. 2004; Ringwalt et al. 2004, 2003). In addition, myriad other factors may influence the need to adapt an EBP, such as financial limitations, agency capacity, funding requirements, or an inhospitable political climate. A host of these issues have been discussed in detail elsewhere (e.g., Fagan and Mihalic 2003; Mitchell et al. 2002; Mowbray et al. 2003). In this article, we focus on issues related to implementation by practitioners once an innovation has been selected for a particular setting. With little guidance provided on how to adapt programs, practitioners may view it as too difficult to implement EBPs even when sufficient resources are available, or EBPs may be implemented in a manner that compromises their effectiveness (e.g., insufficient dosage of services) (Drake et al. 2001; Torrey et al. 2001; Mowbray et al. 2003). Furthermore, dissemination of EBPs may be hindered if they appear to be irrelevant to a specific target population based on surface characteristics of the intervention (Resnicow et al. 2000). For example, a program for unemployed middle-class workers, who may be out of work for the first time in their lives, may not appear applicable to a population of poor women with limited prior work experience.

To address these issues we propose Planned Adaptation, an approach that combines current prevention knowledge about program effectiveness, such as the need to adhere to core program components, with the practical needs of service providers. Core components are "those elements of a program that fundamentally define its nature" and account for the program's effects, as determined by theory, a logic model, or from supporting empirical evidence (Backer 2001). Planned Adaptation is a guide for adapting theory-based EBPs that directs practitioners to consider how population differences may relate to the content of program activities and the theory of change.

\section{Planned Adaptation as a Component of the Integrated Systems Framework}

As suggested by Wandersman and others (Backer 2000; Wandersman et al. 2007), specific tools to support the use of innovations must be provided to practitioners because simply expecting widespread adoption based on research evidence supporting intervention effectiveness is not enough to promote and sustain successful program implementation. One way to bridge the practice-research gap and promote wider dissemination of EBPs is to capitalize on practitioner knowledge in the implementation process to ensure "fit" between the program and the target populations by aiding the process of adaptation. Planned Adaptation provides one capacity-building tool in the Prevention Support System to promote dissemination by providing support in the adaptation process. Planned Adaptation makes it feasible for practitioners to imagine how interventions developed in different contexts might be relevant to their target population, thus potentially broadening the reach of many EBPs.

In addition, Planned Adaptation directs researchers to facilitate the process of program adaptation by requiring that they identify core program components as part of research dissemination (Backer 2001). This work, conceptualized as taking place within the Prevention Synthesis and Translation System, provides a vital foundation for subsequent adaptations by facilitating their adherence to key program protocols, which is known to produce positive outcomes associated with EBPs (Mowbray et al. 2003). Although researchers have made significant progress in using research evidence to identify core program mechanisms, ${ }^{1}$ many EBPs still provide limited empirical evidence identifying important mechanisms of change, particularly with regard to mediating and moderating effects. In the event that a program does not provide empirical evidence that identifies core mechanisms, program theory or a program logic model may also help to elucidate program core components (Backer 2001).

A basic tenet of the ISF is that effective dissemination requires an exchange between researchers and practitioners. Planned Adaptation is a tool within the Prevention Support System that links the Prevention Support System with the Prevention Delivery System. Planned Adaptation provides support at multiple stages of an EBP's dissemination: it serves to orient the provider to the intervention and its theoretical underpinnings; it helps providers identify the

\footnotetext{
${ }^{1}$ While there remains a great need for further progress by prevention researchers in measuring and testing more complex models that clearly point to change-producing mediating and moderating mechanisms, there are a number of initiatives that identify certified EBPs in a range of research areas. Many of these programs, though not all of them, provide empirical evidence on core program components. The following list includes a selection of such initiatives: (1) Blueprints for Violence Prevention Model and Promising Programs (http://www.colorado. edu/cspv/blueprints/); (2) SAMSHA effective substance abuse and mental health programs (http://modelprograms.samhsa.gov/template cf.cfm?page=model_list); (3) Center for Healthy Aging model health program for communities (http://www.healthyagingprograms.org/ content.asp?sectionid=30); (4) Social Programs that Work (http:// www.evidencebasedprograms.org/); and (5) Evidence-based Associates (http://www.evidencebasedassociates.com/programs/).
} 
usefulness of an intervention model for a particular setting; it serves as a framework to orient practitioners to important issues inherent in implementing and adapting EBPs; in addition, it provides some initial direction in developing evaluation strategies. Planned Adaptation is best used in conjunction with additional capacity building services such as program-specific implementation training and ongoing technical support, ideally provided in close consultation with those in the Synthesis and Translation System.

\section{The Practitioner-Researcher Link}

We propose that that the collaboration between researchers and practitioners can take a number of forms. At a minimal level, researchers in the Synthesis and Translation System should provide manuals articulating program activities, with explanation of how activities relate to the program theory. Ideally this information would be based on empirical evidence pointing to the effectiveness of these core mechanisms in producing change. For interventions that have not been tested in a manner that examines core mechanisms, program theory or a logic model can be used to support hypotheses relating program activities to core mechanisms of change (Backer 2001). Secondly, as part of EBP dissemination, researchers in the Synthesis and Translation System can provide additional guidance on potential adaptations of the EBP (e.g. describing ways in which the EBP can be adapted and ways in which it should not be adapted or identifying acceptable cultural modifications that do not conflict with the program theory). For example, research on the Home Visiting Nurse program developed by David Olds and colleagues indicated that delivery by well-trained nurses rather than health paraprofessionals produced optimal program effects, thus training of the intervention deliverers constituted a key mechanism of change in this program (Olds 2002; Olds et al. 2004).

Beyond providing information about key program components and adaptation in general, the Synthesis and Translation System can support practitioners in program adaptation by providing technical assistance (Backer 2000; Mitchell et al. 2004), thus becoming part of the Prevention Support System. This may include consulting at the initial stages of adaptation design or involvement in the implementation of the adapted intervention, potentially providing opportunities for further research on the EBP. A researcher's capacity to provide adaptation specific support to practitioners will be limited by the availability of financial and institutional support for such efforts. Thus, developing funding priorities to support technical support for EBP adaptations is another important part of promoting EBP dissemination (Backer 2001).
There are significant challenges to connecting researchers who design EBPs directly with practitioners, particularly when EBPs become widely disseminated. In such cases it becomes less plausible for researchers to provide individual support for each adaptation. Development of documentation for practitioners on the on-going adaptation processes and their effectiveness can provide a first line of technical support. The availability of such documentation can help to distinguish between those widely disseminated interventions that are not supported by empirical evidence and those that have been deemed effective. In instances when EBPs are sold by commercial vendors, responsibility for providing technical support should transfer to the vendor. Technical assistance is then a part of the product, with emphasis placed on successful adaptation and implementation of innovations, rather than simply dissemination of program content. Finally, a third approach to provision of technical assistance for adaptation of EBPs has emerged in the form of government, university, and private technical support systems, which provide community based agencies with technical support for implementation of EBPs (Mitchell et al. 2002).

Regardless of whether and in what form technical support is made available to practitioners for the adaptation of EBPs, it is imperative that EBPs include information about the core components hypothesized to produce desired outcomes. This link between researchers and practitioners is vital for the successful adaptation of EBPs.

\section{Program Theory: A Key Ingredient of Planned Adaptation}

EBPs rely on program theory (Bickman 1987; Chen 1990; Sidani and Sechrest 1999) to delineate the mechanisms responsible for producing desired program outcomes. One of the challenges of moving programs from the Prevention Synthesis and Translation System to the Prevention Delivery System is making explicit the "key ingredients" of program theory that are critical for program success, and identifying those pieces of the program that may be more amenable to adaptation (Elliott and Mihalic 2004). As noted previously, one key requirement is that researchers and others provide this information, based on program theory and logic models that are supported by empirical evidence (see footnote 1; Backer 2001). Practitioners in the Prevention Delivery System must understand the program theory and its connection to program activities in order for programs to maintain effectiveness in new settings; yet there are few mechanisms in place to assure this capacity among service providers.

Planned Adaptation involves examining an EBP's theory to ensure that adaptations are consistent with the 
core mechanisms of change, as articulated by program theory, supporting empirical evidence, or a program logic model, while allowing for modifications that fit with a new population. The program theory is directly linked to the core mechanism(s) of change through program activities. Program activities should be designed to enact core mechanisms and are thus "hardwired" into the program. These components of the program must be implemented with fidelity to maintain program effectiveness. However, other secondary aspects of the program that are not directly linked to the program theory or the core mechanisms may be adapted to meet the needs of different populations. As outlined by Backer (2001), there are a number of potential adaptations that can be made to a program, including: (1) deletions or additions of program components; (2) modifications to the existing program components; (3) changes in the manner or intensity of delivery of program components; and (4) cultural or other modifications required by local circumstances.

\section{The Four Steps of the Planned Adaptation Approach}

The Planned Adaptation approach consists of four steps. Figure 1 presents these four steps as a logic model in relation to the ISF. After presenting the Planned Adaptation process, we apply these steps to the adaptation of the JOBS Program (Caplan et al. 1989) for use with welfare recipients in welfare-to-work job training programs.

\section{Examine EBP Theory of Change}

Select an EBP that produces the outcome of interest. Although many programs have well defined program theories, some do not; in order to effectively adapt an EBP, it is essential that the program have a discernable theory of change. As described in the ISF, those in the Synthesis and Translation System contribute substantially to this process by creating accessible research products such as metaanalyses, technical and research reports, and literature overviews that highlight key elements of program theory and core components, including: (1) the underlying (direct and mediating) causal mechanisms believed to promote positive outcomes and (2) moderators which may enhance or diminish outcomes. If there are a number of different pathways leading to the outcome of interest, researchers should distinguish which are core mechanisms of change and which are secondary pathways. Core mechanisms directly link the program theory with program activities. Secondary pathways often result from the core mechanism of change but are not the central target of the program activities. In order to adapt the program in a way that preserves the active ingredients of the original program theory and maintains program effectiveness, those elements of the EBP related to the core mechanisms of change should not be modified.

\section{Identify Population Differences}

Examine how the original and new target populations diverge in ways that interact with the mechanisms of change and moderators identified in the program theory. A key question is whether specific program processes are generalized across populations. Identifying these potential points of difference can occur through the intersection of practitioner experience about what "works" with their target population in conjunction with support provided by the Synthesis and Translation System and investigation of the research literature. Not all group differences are necessarily important (see discussion of cultural and race/ ethnic differences, below). One must consider how differences might influence the core mechanisms of change observed in step one. Based on practice knowledge and empirical evidence, researchers and practitioners can collaborate in identifying population differences that relate to the program theory and activities, and use that information to guide changes to the modifiable elements of the program. This is likely an iterative process of considering how and why population differences matter, integrating research from the Synthesis and Translation System and practitioners' experiences from the Prevention Delivery System.

\section{Adapt Program Content}

Once core and modifiable elements of the program are identified, along with population differences, program adaptations can be considered in a systematic way. The adapted program will contain the core mechanisms of change indicated by the program theory, while accommodating important characteristics of the new population through adaptations to the modifiable elements of the program. Thus, the adapted program may include changes to activities in a manner that does not compromise core mechanisms of change, additional processes to enhance program outcomes, or new implementation procedures. Part of the adaptation is deciding whether the program will serve the needs of the new population. Decisions regarding how to adapt program content should evolve from collaborative efforts that link individuals within the Synthesis and Translation and the Prevention Delivery systems. If population differences affect core elements of the program theory, then this EBP may not be the right choice for adaptation to the new population. 


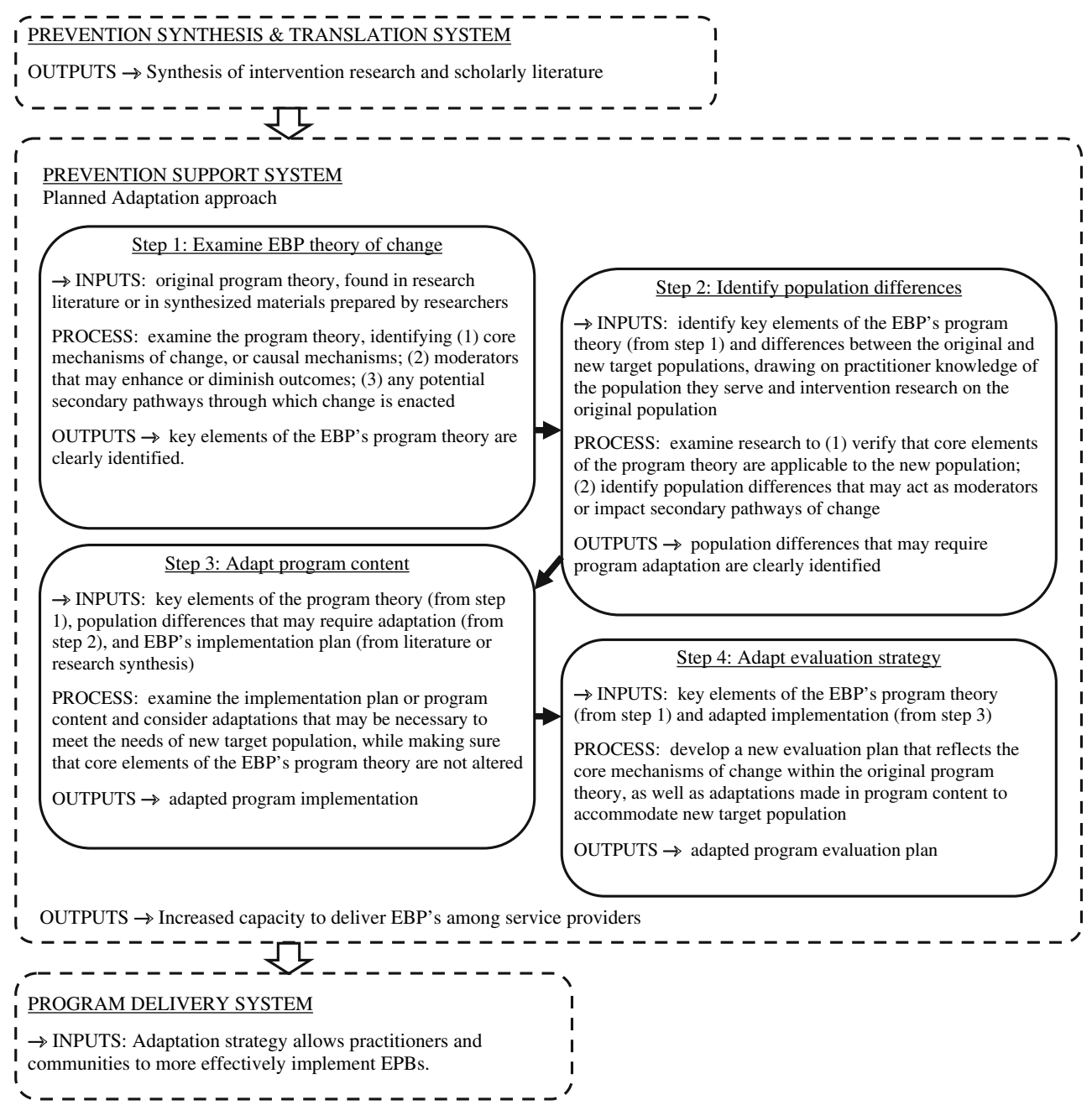

Fig. 1 The Planned Adaptation approach products and processes across the three systems of the interactive systems framework for dissemination and implementation

\section{Adapt Evaluation Strategy}

Although outcome evaluation is not a key component of the ISF, evaluation is a reality in many practice environments and is, therefore, a consideration during program implementation. Because Planned Adaptation promotes a priori consideration of how and why population differences may influence the program before significant adaptations are underway, implementation monitoring and evaluation measurement can be tailored to be consistent with the adapted program, for example, including measurement tools that assess new program components.

\section{The JOBS Program: An Example of Program Adaptation}

We use the example of the JOBS Program to illustrate use of the Planned Adaptation approach for adapting an EBP from one population to another. JOBS (Caplan et al. 1989) was designed as a job-search skill enhancement workshop to promote reemployment among recently unemployed, working- and middle-class individuals. JOBS has been tested effective in a number of different trials (Price et al. 1992; Vinokur et al. 1995; Vinokur and Schul 1997; Vinokur et al. 2000) and has a significant empirical record supporting the direct and mediating processes of the program that led to employment. However, JOBS is not widely used with more "hard-to-serve" unemployed populations such as welfare recipients. Like many EBPs, JOBS provides an example of the difficulty in moving interventions out of the Synthesis and Translation System to widespread dissemination.

As an exception to this general rule, following 1996 federal welfare reform human service administrators in an urban, Eastern county sought to use JOBS with clients in their welfare-to-work program. In this case, there was evidence of organizational capacity to carry out the 
innovation. Job training programs were mandated for welfare recipients in this county. Significant state funds were available for the purpose of carrying out the program and at least one top-level administrator within the organization was invested in the program. However, given that JOBS had been tested with working- and middle-class individuals and the new target population consisted of lowincome women transitioning from welfare to work, issues related to program adaptations needed to be considered. In the remainder of this section we use Planned Adaptation to adapt the JOBS EBP for a new population of low-income women in welfare-to-work programs.

We focus on how differences between the original and new target populations relate to the program theory and pathways of change. Are the core mechanisms underlying the program applicable to a new population that differs in socioeconomic status, psychological functioning, and the type and number of barriers faced in the transition to work? What implications might these population differences have for both the core mechanisms and program content?

Using the Planned Adaptation approach, we first examine the JOBS program theory. Second, we identify key differences between the two populations. In examining population differences we focus on two factors that are hypothesized to be relevant based on research from the JOBS Program: chronic depression and barriers to work. Third, we determine whether JOBS' core mechanisms are applicable to the new population. Several changes to the program content are proposed to increase program effectiveness with welfare recipients. Finally, we consider how the modifications relate to the evaluation of the JOBS Program in this new context (see Table 1).
Examine EBP Theory of Change

JOBS was based on two core mechanisms to promote positive employment outcomes. The first mechanism was promoting participants' self-efficacy, which refers to positive beliefs about the ability to control one's environment (Bandura 1994). JOBS focused on helping participants to regain a sense of self-efficacy with regard to finding a job following the setback of job loss. Self-efficacy beliefs in a specific domain are known to increase coping behavior and reduce perceptions of stress in that domain (Bandura 1989, 1994), as well as to increase perseverance in the face of obstacles (Bandura and Cervone 1983).

The second mechanism of change, inoculation against setbacks, was hypothesized to promote job search persistence by preparing participants to cope with the stress of further setbacks in their search for employment (Vinokur and Schul 1997; Vuori and Vinokur 2005). In the JOBS Program, inoculation against setbacks involved: (1) anticipating problems likely to occur in the job search; (2) developing a course of action related to those problems; and (3) practicing that plan (e.g., role playing) to prepare for the situation.

JOBS sessions were co-led by two group facilitators with approximately 10-15 participants. Five 4-h sessions over the course of 1 week addressed topics such as: "discovering your job skills," "coping with obstacles to employment," "finding job openings and networks," "résumés, contacts, and interviewing," and "the complete interview and planning for setbacks." JOBS used active learning activities such as role-playing interviews with potential employers and brain-storming about how to use social networks to gain new job leads.

Table 1 Summary of the JOBS program planned adaptation

Adaptation stage Target processes

1. Examine EBP program theory

The JOBS intervention uses two core mechanisms to promote employment among participants. The first promotes positive job-search behaviors by increasing participants' self-efficacy in the job search domain. The second mechanism of change promotes persistence in the job-search by inoculating participants against setbacks that they might experience in the job search process.

2. Identify population differences

The original JOBS intervention assumed that participants' primary barriers in re-attaching to the labor market were related to motivating job search behavior. Welfare recipients face unique barriers in the transition to work. High levels of chronic depression may dampen the effectiveness of the intervention in increasing self-efficacy, and consequently limit reemployment outcomes. Furthermore, welfare recipients face challenges such as lack of transportation, child-care issues, and difficulties balancing work and family as single parents.

3. Adapt program content

The population differences point to several adaptations that maintain JOBS' core theory but will bolster effectiveness of the intervention with a low-income female population, including the addition of program content addressing: (1) barriers to work beyond job search behaviors; and (2) coping with chronic depression.

4. Adapt evaluation strategy

The program evaluation strategy should include measurement of competencies gained through new program components. Furthermore, in a program process model depression is theorized to moderate the effects of JOBS on re-employment and it may be embedded within other work barriers that hinder the transition to work. 
The positive effects of the JOBS Program were demonstrated in efficacy trials conducted with working- and middle-class program participants seeking unemployment benefits. JOBS participants became re-employed more quickly and obtained higher quality employment (Caplan et al. 1989; Price et al. 1992; Vinokur et al. 1995, 2000; Vinokur and Schul 1997). Inoculation against setbacks helped participants persist, particularly if they experienced another job loss (Vinokur and Schul 1997). Studies demonstrated that inoculation against setbacks and self-efficacy worked conjointly as key mediators to promote positive reemployment outcomes and lessen depression resulting from unemployment (Vuori and Vinokur 2005).

The JOBS Program has produced similar positive results in sites throughout the world (Vuori et al. 2002; Vuori and Vinokur 2005), and was also adopted for use as a welfareto-work training program in one urban county following welfare reform. The 1996 Personal Responsibility and Work Reconciliation Act (PRWORA) (P.L. 104-193) legislation mandated that most welfare recipients, even those with young children at home, work or engage in work-related activities such as welfare-to-work job training programs in order to receive welfare benefits. The primary goal of welfare-to-work programs is to encourage rapid attachment to the labor market, and JOBS was attractive because of its proven success in promoting attachment to the labor market.

\section{Identify Population Differences}

Broadly speaking, the relevance of the JOBS Program for a low-income population was supported by research indicating that positive psychological mechanisms, including self-efficacy, are related to lowered reliance on welfare, positive employment outcomes, and well-being among low-income women (Danziger et al. 2001; Kalil et al. 2001; Kunz and Kalil 1999). In several cross-sectional studies of low-income mothers working or on welfare, selfefficacy was positively correlated with employment (Jackson 2000; Jackson and Scheines 2005). In a study of current welfare recipients, higher levels of self-efficacy were linked to positive employment gains 4-months later (Lee and Vinokur 2007). Given that the construct of selfefficacy is related to well-being and employment among low-income women, this suggests that the core mechanisms - using inoculation against setbacks to promote selfefficacy-are applicable to welfare recipients transitioning to work.

\section{Depression Among Welfare Recipients}

While evidence points to the relevance of core program mechanisms for a new population of low-income women, there was reason to consider the role of depression within a welfare-to-work population. As pointed out by Price and colleagues (1992), some unemployed people may be too disabled by mental health problems to benefit from a shortterm intervention that does not directly target decreasing depression. As a result, in the original JOBS intervention potential participants who had clinical levels of depression were excluded from participating (Vinokur and Schul 1997). Although JOBS' activities did not focus on depression explicitly, the program had direct and indirect effects in preventing depression brought about by sudden job loss among those that did participate (Vinokur and Schul 1997; Vuori and Vinokur 2005). In contrast, in the welfare setting, women applying for welfare benefits are almost always mandated to participate in welfare-to-work job training programs regardless of their mental health status.

Depression may be a factor that complicates the implementation and effectiveness of JOBS with welfare recipients. Low-income women experience clinical depression at rates that exceed the national average (Kessler et al. 1994). In a representative sample of current and former welfare recipients $35 \%$ met the diagnostic criteria for major depression and an additional $25 \%$ reported symptoms of major depression within the last year (Danziger et al. 2000). Estimates indicate that $30-40 \%$ of the welfare population have a mental health problem, including depression (Coiro 2001; Corcoran et al. 2004; Chandler et al. 2005; Taylor and Barusch 2004; Vinokur 2003).

Research also suggests that the mechanisms related to the onset of depression differ among low-income women. Working-and middle-class individuals are at increased risk of experiencing a depressive episode as a result of unemployment (Dooley et al. 1994; Kessler et al. 1987, 1989), while living in poverty is linked to depression through other mechanisms. Poverty increases the likelihood of early onset of depression (Kessler 1997; Siefert et al. 2000; Belle and Doucet 2003), and early onset is associated with lowered threshold for subsequent depressive episodes (Kendler et al. 2000). One study of low-income mothers found that repeated or extended episodes of depression increased the risk for welfare dependence (Lehrer et al. 2002). Depression for women in a low-income context is linked to complex factors that extend beyond sudden and unexpected unemployment (Belle and Doucet 2003; Kendler et al. 2000; Kessler 1997; Lehrer et al. 2002; Siefert et al. 2000).

An implication is that, in contrast to results found in previous studies with working- and middle-class individuals, low income women have unique vulnerabilities that may compromise the potential salubrious mental health benefits of participation in a short-term program like JOBS. However, the program may retain its effectiveness for 
women who are experiencing challenges specific to their employment situation or for those experiencing situational depression (Kalil et al. 2001; Lehrer et al. 2002; Vinokur 2003). In sum, in the welfare context, pre-existing levels of depression may serve as a moderator of program effects. Higher levels of initial depression are likely to dampen gains in self-efficacy and likelihood of subsequent re-employment. Depressed women may need more intensive and targeted services (Lee 2005) than those provided by a short-term program. A related hypothesis is that mental health gains may be short-lived among those who begin the JOBS program with high or clinical levels of depression. Thus, an adapted program model should imbed depressive symptomatology within the constellation of work barriers that low-income women face as they transition to work (Lee and Vinokur 2007).

\section{Barriers to Work Among Welfare Recipients}

An assumption underlying the original JOBS program is that participants' primary barriers to re-employment were motivating and sustaining effective job search behaviors. Evidence suggests that mobilizing effective job search behaviors are secondary for welfare recipients who differ from middle-class individuals in the source and number of barriers to employment. Many of the original JOBS participants were educated with established work experience, and the majority were married (Caplan et al. 1989; Vinokur, Price and Caplan 1996). In contrast, women on welfare are often single parents with limited labor market experience, low levels of education, and fewer job skills (Danziger et al. 2000; Vinokur 2003).

Beyond human capital variables, the majority of welfare recipients face additional obstacles to employment, including mental and physical health problems (Chandler et al. 2005; Corcoran et al. 2004; Taylor and Barusch 2004), lack of transportation (Danziger et al. 2000; Vinokur 2003), and challenges managing work and family responsibilities while trying to find employment (Lee 2007). The jobs available to welfare recipients are often inconsistent with the demands of parenting, and nonstandard work hours may constrain childcare options (Danziger et al. 2004; Scott et al. 2005; Roy et al. 2004). In one study, $31 \%$ of current and former welfare recipients reported lacking adequate and affordable childcare (Danziger et al. 2004). Low-income mothers also have fewer protective elements to decrease work-family conflict, such as a supportive supervisor, a spouse, or a high-quality job (Eby et al. 2005; Erdwins et al. 2001; Parasuraman and Greenhaus 2002).

Research has documented that barriers to work significantly hinder the welfare to work transition. Women with more work barriers work less over time and have more difficulty sustaining employment (Corcoran et al. 2004; Danziger et al. 2000; Zedlewski 2003). Barriers decrease job retention and increase the likelihood that a former recipient will return to welfare (Loprest 2002; Nam 2005). Consequently, in order to optimize effectiveness, programs targeting welfare-to-work populations must address the wide range of work barriers that extend beyond lack of job search skills experienced by low-income women.

\section{Adapt JOBS Program Content}

Based on the identification of population differences that exist between the welfare-to-work and the original JOBS populations, we identified program modifications intended to optimize the effectiveness of the JOBS Program in this new context, while maintaining consistency with the core program theory identified in step one.

\section{Depression Among Welfare Recipients}

As noted above, the onset, nature, and duration of depression differs as a function of socio-economic status and gender. Therefore, program content explicitly focused on promoting women's ability to cope with a wide range of employment-related stressors may be beneficial for mental health. Introduction of content focusing on basic problem solving and stress management skills extending beyond job search issues is consistent with the theoretical focus on self-efficacy, and likely to be to useful to women who face a variety of life stressors. For example, in the original JOBS program, participants brainstorm about use of their social network to find employment; in the adapted program, use of social networks might be construed more broadly in terms of both gaining social support in a way that extends beyond the job search in addition to using social networks to find employment.

\section{Barriers to Work Among Welfare Recipients}

Because of the additional barriers faced by the welfare to work population and the negative consequences of these barriers on employment outcomes, an intervention that seeks to connect welfare mothers to the labor force must acknowledge the challenges associated with managing work and family in a low-income context. This points to the need for program content that extends beyond the original program's focus on job search skills per se (e.g., contacting employers, interviewing skills, résumé writing). Adapted program activities thus assess and provide strategies for coping with and ameliorating common work barriers, such as conflict between work and parenting, challenges to finding adequate day care, and transportation issues, which are highly relevant to low-income mothers. 
From this perspective, activities using the inoculation against setbacks framework build on a broader range of experiences. Participants identify barriers to work that they are likely to experience in an employment setting, such as transportation and childcare problems. Participants build on the strengths of other group members and learn how others have addressed these problems, then develop an individual plan of action for what to do when this problem arises. Finally, they use active techniques such as role playing to put their plan into action.

These adaptations are consistent with the theory underlying the inoculation against setbacks mechanism, but increase the relevance of the program to low-income mothers. The goal is to maintain the core process by which inoculation against setbacks promotes change and promotes self-efficacy, while altering the content to address relevant work barriers. These concrete skills focusing on anticipation of and strategizing with regard to work barriers are likely to promote self-efficacy in the face of job-search and work-family barriers to employment.

\section{Adapt Evaluation Strategy}

Program adaptation provides an opportunity to understand how programs function in different contexts, and over time, the evidence regarding specific program innovations and adaptations will illuminate what works best with specific populations and under what circumstances. Obtaining such evidence necessitates adjustment of the evaluation strategy. In this example, measurement should expand beyond assessment of depressive symptoms to include potential proximal indicators of well-being such as coping ability, mood, and perceived daily stressors. Furthermore, measurement of perceived work barriers and participants' strategies for coping with barriers may reveal that the program reduces the extent to which participants perceive work barriers as impeding their employment options.

Preliminary non-experimental evidence from the JOBS welfare-to-work program suggested that participants had high rates of re-attachment to the labor force following the intervention (Vinokur and Lee in preparation). Pre- and post-test data indicated that rates of depression were lessened and participants experienced increased self-efficacy; however, it is not known whether initial level of depressive symptoms moderated program effects. As a caveat, it is important to note that outcomes were not directly linked to any program modifications; therefore, given the limitations of the evaluation strategy these outcomes cannot be linked to specific program content (e.g., content addressing work barriers). What the preliminary results suggest is that the JOBS model is appropriate for implementation in a welfare-to-work context. Future research is needed to more carefully examine mediators and moderators of program effects using a more rigorous study design.

\section{A Special Case of Program Adaptation}

One objective of disseminating EBPs is to provide programs with proven success to populations in need of effective services. Some have pointed to the need for cultural adaptations when serving distinct cultural or racial/ ethnic groups (e.g. Castro et al. 2004). However, there is mixed evidence regarding the need for and the success of cultural adaptations of intervention programs (Hecht et al. 2003; Pilgrim et al. 2006). While cultural or racial/ethnic specific adaptations may be necessary, these adaptations should be guided by program theory, as suggested by the Planned Adaptation approach. The Planned Adaptation approach intends to move beyond surface structure issues (Resnicow et al. 2000), for example, maintaining cultural sensitivity through matching content of intervention materials or intervention deliverers on observable characteristics of the population being targeted. Instead, the focus is on promoting a more systematic understanding of "deep structure" issues, such as addressing whether cultural factors are indeed central to the core mechanism of change. In this view, adaptations to local circumstances are appropriate as long as they do not contradict aspects of the program theory that provide the "cognitive blueprint" for action (Price et al. 1998).

\section{Program Adaptation Builds Capacity at Multiple Levels}

As highlighted by the ISF (Wandersman et al. 2008), research is often difficult for practitioners to interpret; individuals, organizations, and communities sometimes lack the capacity to implement and sustain innovations; and common dissemination practices often presume that practitioners are passive recipients of an innovation rather than active participants in the dissemination and implementation process. Planned Adaptation links the Prevention Synthesis and Translation System and the Prevention Delivery System by proposing that practitioners and community members are active participants in the knowledge development and intervention translation processes. It suggests that practitioners and community members have a wealth of information regarding the populations most in need of EBPs, and that those needs must be taken into consideration in program implementation. Furthermore, Planned Adaptation assumes that practitioners are interested in the research process and wish to gain greater understanding of the programs (and 
program theories) that will best serve the communities they care about. Indeed, our teaching and practice experiences suggest that many human service providers are very interested in participating in this process and there is great need for "practice-based research" to facilitate bridging the research-practice gap.

Within the Prevention Support System, Planned Adaptation provides support for innovation implementation. Planned Adaptation may serve as a diagnostic tool to examine the capacity of a service agency. If an agency or a few key individuals within an agency are not able to engage in the first steps of the Planned Adaptation process, such as identifying the program theory or examining potential points of difference between the target population and the population the intervention was developed with, this agency may not have adequate capacity to implement the program and will require additional support. Within the Prevention Synthesis and Translation System, Planned Adaptation calls on researchers to extend their innovation development to include specific guidelines for how to adapt the innovation in different settings and with different populations.

\section{Program Adaptation across the Interactive Systems Framework}

In our conceptualization, Planned Adaptation is best suited to take place in the Prevention Support System, utilizing inputs from the Prevention Synthesis and Translation System and the Prevention Delivery System. Ideally, program adaptation is planned before implementation in a new context, utilizing the knowledge and expertise of practitioners and researchers. However, there are likely to be instances when adaptation occurs at all levels of the ISF. Thus, it is important to consider how Planned Adaptation might play out within all three IFS Systems.

For example, within the Prevention Synthesis and Translation System, prevention scientists and other researchers may test EBPs with new populations and subgroups (e.g., August et al. 2006; Botvin et al. 2003; Gottfredson et al. 2006). As such, some may decide to develop adaptations in a manner that is consistent with the guidelines identified in this article, but with less direct input from other systems of the ISF. There are likely to be a number of potential strengths and weaknesses when adaptations occur primarily within the Prevention Synthesis and Translation System. A clear strength is that researchers should be well-equipped to use the research literature to identify key mechanisms of change, to consider how population differences might be related to these mechanisms, and to modify the program accordingly. However, a potential drawback to adaptation that occurs primarily or solely within the Prevention Synthesis and Translation System is that it may also entail less direct input and involvement from those within the Prevention Delivery System-specifically, practitioners and other community members. Thus, the resulting program, whether adapted or in its original form, may not be well informed regarding the needs of clients and the realities of program implementation in a particular context, information that is more readily available within the Prevention Delivery System. Consequently, programs adapted in this manner will not be immune to the limitations (e.g., lack of infrastructure to support innovations) that affect implementation of interventions more generally. Equally problematic from the perspective of the practitioner may be lack of knowledge regarding how and why certain adaptations were implemented, and little ensuing understanding of the theoretical foundation of the program. All of these factors may hinder effective implementation of the adapted program.

Perhaps more common are adaptations that occur "after the fact" in the Prevention Delivery System. It is not uncommon for practitioners and other community members to implement new and interesting EBPs and make changes that fundamentally compromise the integrity of the program (Mowbray et al. 2003). For Planned Adaptation to occur effectively in the Prevention Delivery System, practitioners must have access to research and scholarly literature that synthesizes and translates the core components of the program. Provided with a sense of how population differences might influence the program theory and core components they can make adaptations and develop an evaluation strategy that meets the needs of their population. As highlighted by the ISF, practitioners should be supported in this process by consultants well versed in prevention interventions and their adaptation.

While Planned Adaptation that occurs primarily within the Prevention Delivery System may more accurately reflect community resources and constraints, some potential drawbacks are worth considering. First, consistent with the ISF, a goal of Planned Adaptation is to provide a framework that promotes the interaction of researchers and practitioners, so that these perspectives can be used to bridge the science-practice gap. Therefore, adaptations that take these multiple perspectives into account are likely to be the most effective and also the most instructive in terms of future research and theory development. Adaptations implemented solely within the Prevention Delivery System are likely to lack this multiple-systems perspective. Without input from other systems, adaptations within the Prevention Delivery System are more likely to violate core program components, leading to problems associated with lack of fidelity in program implementation, which are well documented (Mowbray et al. 2003). 


\section{Implications for the Interactive Systems Framework}

Planned Adaptation has a number of implications for the ISF. Planned Adaptation makes explicit connections within and between the systems of the ISF. First, Planned Adaptation is intended to build innovation-specific capacity within the Prevention Support System, because the model emphasizes the need for researchers and practitioners to collaborate in deciding what innovations are best suited for a particular population or context, and how to implement an innovation in a manner that facilitates its sustainability.

Second, Planned Adaptation intends to promote the accessibility of science-based programs to practitioners in a manner that places responsibility for program adaptation on both practitioners and researchers, and assumes that both researchers and practitioners benefit from active collaboration in the adaptation process. As suggested by the Synthesis and Translation System, those who develop and test interventions should synthesize and translate research in a manner that is accessible to practitioners and be available to practitioners as consultants or advisors in the adaptation process. Practitioners are valid in their desire to use programs that they are confident will fit with the needs of the populations they serve, and practitioner knowledge should be a critical aspect of program dissemination and implementation.

As an example of how this might take place, the social work paraprofessionals who led the welfare-to-work version of the JOBS program participated in an initial training period supplemented by ongoing "booster shot" sessions led by a "master" JOBS trainers. This provided multiple opportunities for an ongoing exchange between the researchers, practitioners, and the welfare administrators funding the program, to address questions related to program implementation. The master trainer was available to provide feedback on the content and type of adaptations that were appropriate. While in a more rigorous research setting an ongoing exchange of this type may compromise the research protocol, this was done in the context of an already established, manualized intervention. Furthermore, early training sessions focused on helping paraprofessionals to understand the theoretical underpinnings of JOBS, thus they had greater capacity to implement adaptations that were consistent with the program theory. Monitoring and ongoing paraprofessional training served to facilitate program fidelity.

Further revisions of the ISF should explicitly indicate practitioners' roles in the Synthesis and Translation System and state additional responsibilities that researchers have for participating in the Prevention Delivery System. Related to this is the need for increasing knowledge accessibility beyond synthesis and translation. Metaanalysis papers and literature reviews are helpful but they are still often impenetrable by service providers. Furthermore, they tend to narrowly address one issue or topical area. It is not common for meta-analyses to connect across key intervention components, such as the relationship between program theory, program activities, and population differences. Researchers should clearly identify the core mechanisms of change and the modifiable components of an intervention, and provide guidance on how these processes might differ as a function of context. Furthermore, researchers should clearly elaborate how program activities and protocols relate to the program theory, and present information regarding best practices for intervention delivery. Related to this is the need for more attention to guiding appropriate evaluation and measurement, an aspect of dissemination that receives little attention. As a necessary extension of these developments, and as pointed out by Wandersman et al. (2008), research journals (and other venues) should publish a range of papers that address intervention delivery that are written in a language accessible to practitioners.

The Planned Adaptation approach also points to the need to think more broadly about fidelity within the ISF. Program implementation with perfect fidelity is not likely in service settings, particularly when the desire is to serve a population rather than test a theoretical model. Fidelity discussions tend to center around the need to adhere to manuals and standardized protocols of intervention delivery. Too little attention is given to the role of program theory, and specifically mechanisms to ensure that practitioners can deliver program activities as outlined in a manual and also understand the link between those activities and the program theory of change. When practitioners understand the program theory, they are better able to adapt the program in ways that does not violate program fidelity. Again, as noted above, little attention is given to helping practitioners think about ways to measure implementation fidelity or to selecting measurement tools for outcome evaluation. Other ways of thinking about and measuring fidelity should be included within the training elements of the Prevention Support System.

\section{Conclusion}

One of the central goals of the ISF is to increase the use of existing research knowledge (Wandersman et al. 2008). Planned Adaptation serves as one tool in the Prevention Support System toolkit to promote wider dissemination of EBPs. Consistent with the broader goals of the ISF, Planned Adaptation calls upon researchers to engage with communities to disseminate research findings that are relevant and usable by practitioners. Furthering, following from the Planned Adaptation approach, practitioners must 
consider the "theory" in the programs they deliver so that they are properly implemented and evaluated. This process involves considering how characteristics of their target population might interact with the core mechanisms of change in an intervention. Ultimately, adapting programs to new target populations while also maintaining program integrity is an opportunity to bridge science and practice as practitioners and researchers gain information about the unique needs of sub-populations and consider how these needs impact and change interventions. Adaptation that evolves out of an exchange between practitioners and researchers will push the field of prevention science forward as researchers learn more about new and varied practice settings and examine the generalizability of theory-based programs in different contexts, and practitioners have the opportunity to effectively deliver EBP to their clients.

Acknowledgement The first author was supported by NIMH training grant T32-MH63057 during preparation of this manuscript. An earlier version of this paper was presented at a symposium of the 2004 Society for Social Work and Research (SSWR) annual meeting, New Orleans, LA. We would like to thank Abe Wandersman, Amiram D. Vinokur, Daphna Oyserman, and two anonymous reviewers for helpful comments on earlier drafts of this paper.

\section{References}

August, G. J., Bloomquist, M. L., Lee, S. S., Realmuto, G. M., \& Hektner, J. M. (2006). Can evidence-based prevention programs be sustained in community practice settings? The Early Risers' advanced-stage effectiveness trial. Prevention Science, 7, $151-165$.

Backer, T. E. (2000). The failure of success: Challenges of disseminating effective substance abuse prevention programs. Journal of Community Psychology, 28, 363-373.

Backer, T. E. (2001). Finding the balance: Program fidelity and adaptation in substance abuse prevention: A state-of-the-art review. Rockville, MD: Department of Health and Human Services, Substance Abuse and Mental Health Services Administration.

Bandura, A. (1989). Human agency in social cognitive theory. American Psychologist, 44, 1175-1184.

Bandura, A. (1994). Self-efficacy. In V. S. Ramachaudran (Ed.), Encyclopedia of human behavior (Vol. 4, pp. 71-81). New York: Academic Press. (Reprinted in H. Friedman (Ed.) (1998). Encyclopedia of mental health. San Diego: Academic Press).

Bandura, A., \& Cervone, D. (1983). Self-evaluative and self-efficacy mechanisms governing the motivational effects of goal systems. Journal of Personality and Social Psychology, 45, 1017-1028.

Belle, D., \& Doucet, J. (2003). Poverty, inequality, and discrimination as sources of depression among US women. Psychology of Women Quarterly, 27, 101-113.

Bernal, G., \& Sáez-Santiago, E. (2006). Culturally centered psychosocial interventions. Journal of Community Psychology, 34, 121-132.

Bickman, L. (1987). The functions of program theory. New Directions for Program Evaluation, 33, 5-17.

Blakely, C. H., Mayer, J. P., Gottschalk, R. G., Schmitt, N., Davidson, W. S., Roitman, D. B., et al. (1987). The fidelity-adaptation debate: Implications for the implementation of public sector social programs. American Journal of Community Psychology, $15,253-268$.

Botvin, G. J., Schinke, S. P., Epstein, J. A., \& Diaz, T. (2003). Effectiveness of culturally focused and generic skills training approaches to alcohol and drug abuse prevention among minority youths. Psychology of Addictive Behaviors, 8, 116-127.

Caplan, R. D., Vinokur, A. D., Price, R. H., \& van Ryn, M. (1989). Job seeking, reemployment, and mental health: A randomized field experiment in coping with job loss. Journal of Applied Psychology, 74, 759-769.

Castro, F. G., Barrera, M., \& Martinez, M. (2004). The cultural adaptation of prevention interventions: Resolving tensions between fidelity and fit. Prevention Science, 5, 41-45.

Chandler, D., Meisel, J., Jordan, P., Menees-Rienzi, B., \& Goodwin, S. N. (2005). Mental health, employment, and welfare tenure. Journal of Community Psychology, 33, 587-609.

Chen, H. T. (1990). Theory-driven evaluation. Newbury-Park, CA: Sage.

Coiro, M. J. (2001). Depressive symptoms among women receiving welfare. Women and Health, 32, 1-23.

Corcoran, M., Danziger, S. K., \& Tolman, R. M. (2004). Long term employment of African-American and white welfare recipients and the role of persistent health and mental health problems. Women \& Health, 39, 21-40.

Danziger, S. K., Ananat, E. O., \& Browning, K. G. (2004). Childcare subsidies and the transition from welfare to work. Family Relations, 53, 219-228.

Danziger, S. K., Corcoran, M., Danziger, S., Heflin, C., Kalil, A., Levine, J., et al. (2000). Barriers to employment of welfare recipients. In: R. Cherry \&W.M. Rodgers III (Eds.), Prosperity for all? The economic boom and African Americans (pp. 245278). New York: Russell Sage Foundation.

Danziger, S. K., Carlson, M. J., \& Henly, J. R. (2001). Post-welfare employment and psychological well-being. Women \& Health, $32,47-49$.

Dooley, D., Catalano, R., \& Wilson, G. (1994). Depression and unemployment: Panel findings from the Epidemiologic Catchment Area study. American Journal of Community Psychology, $22,745-765$.

Drake, R. E., Goldman, H. H., Leff, H. S., Lehman, A. F., Dixon, L., Mueser, K. T., et al. (2001). Implementing evidence-based practices in routine mental health service settings. Psychiatric Services, 52, 179-182.

Eby, L. T., Casper, W. J., Lockwood, A., Bordeaux, C., \& Brinley, A. (2005). Work and family research in IO/OB: Content analysis and review of the literature (1980-2002). Journal of Vocational Behavior, 66, 124-107.

Elliott, D. S., \& Mihalic, S. (2004). Issues in disseminating and replicating effective prevention programs. Prevention Science, 5 , $47-53$.

Erdwins, C. J., Buffardi, L. C., Casper, W. J., \& O'Brien, A. S. (2001). The relationship of women's role strain to social support, role satisfaction, and self-efficacy. Family Relations, 50, 230-238.

Fagan, A. A., \& Mihalic, S. (2003). Strategies for enhancing the adoption of school-based prevention programs: Lessons learned from the Blueprints for Violence prevention replications of the Life Skills Training Program. Journal of Community Psychology, 31, 235-253.

Fraser, M. W. (2004). Intervention research in social work: Recent advances and continuing challenges. Research on Social Work Practice, 14, 210-222.

Gira, E. C., Kessler, M. L., \& Poertner, J. (2004). Influencing social workers to use research evidence in practice: Lessons from medicine and the allied health professions. Research on Social Work Practice, 14, 68-79. 
Gottfredson, D., Kumpfer, K., Polizzi-Fox, D., Wilson, D., Puryear, D., Beatty, P., \& Vilmenay, M. (2006). The strengthening Washington DC families project: A randomized effectiveness trial of family-based prevention. Prevention Science, 7, 57-74.

Hecht, M. L., Marsiglia, F. F., Elek, E., Wagstaff, D. A., Kulis, S., Dustman, P., \& Miller-Day, M. (2003). Culturally grounded substance use prevention: An evaluation of the Keepin' it R.E.A.L. curriculum. Prevention Science, 4, 233-248.

Howard, M. O., McMillen, C. J., \& Pollio, D. E. (2003). Teaching evidence-based practice: Toward a new paradigm for social work education. Research on Social Work Practice, 13, 234-259.

Jackson, A. P. (2000). Maternal self-efficacy and children's influence on stress and parenting among single black mothers in poverty. Journal of Family Issues, 21, 3-16.

Jackson, A. P., \& Scheines, R. (2005). Single mothers' self-efficacy, parenting in the home environment, and children's development in a two-wave study. Social Work Research, 29, 7-20.

Kalil, A., Schweingruber, H. A., \& Seefeldt, K. S. (2001). Correlates of employment among welfare recipients: Do psychological characteristics and attitudes matter? American Journal of Community Psychology, 29, 701-724.

Kendler, K. S., Thornton, L. M., \& Gardner, C. O. (2000). Stressful life events and previous episodes in the etiology of major depression in women: An evaluation of the "kindling" hypothesis. American Journal of Psychiatry, 157, 1243-1251.

Kessler, R. C., McGonagle, K. A., Zhao, S., Nelson, C. B., Hughes, M., Eshleman, S., et al. (1994). Lifetime and 12-month prevalence of DSM-III-R psychiatric disorder in the United States: Result from the National Comorbidity Survey. Archives of General Psychiatry, 51, 8-19.

Kessler, R. C., Turner, J. B., \& House, J. S. (1987). Intervening processes in the relationship between unemployment and health. Psychological Medicine, 17, 949-961.

Kessler, R. C., Turner, J. B, \& House, J. S. (1989). Effects of unemployment on health in a community survey: Main, modifying, and mediating effects. Journal of Social Issues, 44, 69-85.

Kessler, R. C. (1997). The effects of stressful life events on depression. Annual Review of Psychology, 48, 191-214.

Kunz, J., \& Kalil, A. (1999). Self-esteem, self-efficacy, and welfare use. Social Work Research, 23, 119-126.

Lee, S. J. (2005). Facilitating the welfare-to-work transition for women with a mental health work barrier. Journal of Human Behavior in the Social Environment, 12, 127-143.

Lee, S. J. (2007). Low-income women and the work-family balancing act. Manuscript submitted for publication.

Lee, S. J., \& Vinokur, A. D. (2007). Work barriers in the context of pathways to the employment of welfare-to-work clients. American Journal of Community Psychology, 40, 301-312.

Lehrer, E., Crittenden, K., \& Norr, K. F. (2002). Depression and economic self-sufficiency among inner-city minority mothers. Social Science Research, 31, 285-309.

Loprest, P. (2002). Snapshots of America's families III, No.3: Disconnected welfare leavers face serious risks. Washington, DC: The Urban Institute.

Mitchell, R. E., Florin, P., \& Stevenson, J. F. (2002). Supporting community-based prevention and health promotion initiatives: developing effective technical assistance systems. Health Education \& Behavior, 29, 620-639.

Mitchell, R. E., Stone-Wiggins, B., Stevenson, J. F., \& Florin, P. (2004). Cultivating capacity: Outcome of a statewide support system for prevention coalitions. Journal of Prevention \& Intervention in the Community, 27, 67-87.

Mowbray, C. T., Holter, M. C., Teague, G. B., \& Bybee, D. (2003). Fidelity criteria: Development, measurement, and validation. American Journal of Evaluation, 24, 315-340.
Nam, Y. (2005). The roles of employment barriers in welfare exits and reentries after welfare reform: Event history analyses. The Social Service Review, 79, 268-293.

Olds, D. L. (2002). Prenatal and infancy home visiting by nurse: From randomized trials to community replication. Prevention Science, $3,153-172$.

Olds, D. L., Robinson, J., Pettitt, L., Luckey, D. W., Holmberg, J., Ng, R. K., Isacks, K., Sheff, K., \& Henderson, C. R. (2004). Effects of home visits by paraprofessionals and by nurses: Age 4 followup results of a randomized trial. Pediatrics, 114, 1560-1568.

Parasuraman, S., \& Greenhaus, J. H. (2002). Toward reducing some critical gaps in the work-family research. Human Resource Management Review, 12, 299-312.

Pilgrim, C. C., Schulenberg, J. E., O’Malley, J. M., Bachman, J. G., \& Johnston, L. D. (2006). Mediators and moderators of parental involvement on substance use: A national study of adolescents. Prevention Science, 7, 75-89.

Price, R. H., Friedland, D. S., Choi, J. N., \& Caplan, R. D. (1998). Job-loss and work transitions in a time of global economic change. In X. B. Arriaga \& S. Oskamp (Eds.), Addressing community problems: Psychological research and interventions (pp. 195-888). Thousand Oaks, CA: Sage Publications.

Price, R. H., van Ryn, M., \& Vinokur, A. D. (1992). Impact of preventive job search intervention on the likelihood of depression among the unemployed. Journal of Health and Social Behavior, 33, 158-167.

Resnicow, K., Soler, R. E., Braithwaite, R. L., Ahluwalia, J. S., \& Butler, J. (2000). Cultural sensitivity in substance use prevention. Journal of Community Psychology, 28, 271-290.

Ringwalt, C. L., Ennett, S., Johnson, R., Rohrback, L. A., SimonsRudolph, A., Vincus, A., et al. (2003). Factors associated with fidelity to substance use prevention curriculum guides in the nation's middle schools. Health Education and Behavior, 30, 375-391.

Ringwalt, C. L., Vincus, A., Ennett, S., Johnson, R., \& Rohrbach, L. A. (2004). Reasons for teachers' adaptation of substance use prevention curricula in schools with non-white student populations. Prevention Science, 5, 61-67.

Rosen, A. (2003). Evidence-based social work practice: Challenges and promise. Social Work Research, 27, 197-208.

Roy, K. M., Tubbs, C. Y., \& Burton, L. M. (2004). Don't have no time: Daily rhythms and the organization of time for low-income families. Family Relations, 53, 168-178.

Scott, E. K, London, A. S., \& Hurst, A. (2005). Instability in patchworks of child care when moving from welfare to work. Journal of Marriage and the Family, 67, 370-386.

Sidani, S., \& Sechrest, L. (1999). Putting program theory into operation. American Journal of Evaluation, 20, 227-238.

Siefert, K., Bowman, P., Heflin, C., Danziger, S., \& Williams, D. (2000). Social and environmental predictors of maternal depression in current and recent welfare recipients. American Journal of Orthopsychiatry, 70, 510-522.

Taylor, M. J., \& Barusch, A. S. (2004). Personal, family, and multiple barriers of long Term welfare recipients. Social Work, 49, $175-184$.

Torrey, W. C., Drake, R. E., Dixon, L., Burns, B. J., Flynn, L., Rush, A. J., et al. (2001). Implementing evidence-based practices for persons with severe mental illnesses. Psychiatric Services, 52, $45-50$.

Vinokur, A. D. (2003). Final evaluation report: The impact of the "From the Ground Up" program and its "Winning New Jobs" workshop on welfare-to-work clients of the Department of Social Services. Baltimore County, MD. University of Michigan: Ann Arbor.

Vinokur, A. D., \& Lee, S. J. (in preparation). Evaluating outcomes of a theory-based job training program for welfare-to-work clients. 
Vinokur, A. D., Price, R. H., \& Caplan, R. D. (1996). Hard times and hurtful partners: How financial strain affects depression and relationship satisfaction of unemployed persons and their spouses. Journal of Personality and Social Psychology, 71, 166-179.

Vinokur, A. D., Price, R. H., \& Schul, Y. (1995). Impact of the JOBS intervention on unemployed workers varying in risk for depression. American Journal of Community Psychology, 23, 39-74.

Vinokur, A. D., \& Schul, Y. (1997). Mastery and inoculation against setbacks as active ingredients in the JOBS intervention for the unemployed. Journal of Consulting and Clinical Psychology, 65, 867-877.

Vinokur, A. D., Schul, Y., Vuori, J., \& Price, R. H. (2000). Two years after a job loss: Long-term impact of the JOBS program on reemployment and mental health. Journal of Occupational Psychology, 5, 32-47.
Vuori, J., Silvonen, J., Vinokur, A. D., \& Price, R. H. (2002). The Työhön Job Search Program in Finland: Benefits for the unemployed with risk of depression or discouragement. Journal of Occupational Health Psychology, 7, 5-19.

Vuori, J., \& Vinokur, A. D. (2005). Job-search preparedness as a mediator of the effects of the Työhön Job Search Intervention on re-employment and mental health. Journal of Organizational Behavior, 26, 275-291.

Wandersman, A., Duffy, J., Flaspohler, P., Noonan, R., Lubell, K., Stillman, L., Blachman, M., Dunville, R., \& Saul, J. (2008). Bridging the gap between prevention science and practice: The Interactive systems framework for dissemination and implementation. American Journal of Community Psychology, 41, 3-4.

Zedlewski, S. R. (2003). Snapshots of America's families III, No.3: Work and barriers to work among welfare recipients in 2002. Washington DC: The Urban Institute. 